\title{
On the Temperature Dependence of the Double Layer Capacitance of Ionic Liquids ${ }^{\dagger}$
}

\author{
Ming Chen ${ }^{\mathrm{a}}$, Zachary A. H. Goodwin ${ }^{\mathrm{b}, \mathrm{c}}$, Guang Feng ${ }^{\mathrm{a}}$, Alexei A. \\ Kornyshev $^{\mathrm{b}, *}$ \\ ${ }^{a}$ State Key Laboratory of Coal Combustion, School of Energy and Power Engineering, \\ Huazhong University of Science and Technology (HUST), Wuhan 430074, China \\ ${ }^{b}$ Imperial College London, Department of Chemistry, Imperial College Rd, London, SW7 \\ $2 A Z$ \\ ${ }^{c}$ Imperial College London, Department of Physics, Imperial College Rd, London, SW
} $2 A Z$

\begin{abstract}
The temperature dependence of room temperature ionic liquids differential capacitance is studied here with both theoretical and computational methods. On the theory front, the lattice-gas mean-field theory of ionic liquids is further generalised to account for ?ion pairing? and ?neutral aggregate? formation. An anomalous temperature dependence of linear response capacitance was found, similar to that reported in earlier work. The theory also predicted that differential capacitance curves transform from a camel to bell shape with increasing temperature. Molecular dynamics simulations verified the expected transition in shape of differential capacitance curves with temperature and the dependence of linear response capacitance on temperature. Further investigation into charge density distributions revealed an ordered structure, reminiscent of oriented ion pairs and neutral aggregates, extending far enough from the electrode to control the capacitance-voltage response. It was found that these structures were dismantled with increasing temperature, as predicted by the mean-field theory.
\end{abstract}

Keywords: room temperature ionic liquids, mean-field, temperature,

\footnotetext{
${ }^{*}$ Corresponding author

Email address: a.kornyshev@imperial.ac.uk (Alexei A. Kornyshev)

$\dagger$ This paper is devoted to the memory of an outstanding electrochemist, and great personality, Roger Parsons, whom one of us (AAK) personally knew and whose contributions to understanding the electrical double layer influenced many researches.
} 
differential capacitance

\section{Introduction}

Room temperature ionic liquids (RTILs), ultra-concentrated electrolytes that are liquid under $100{ }^{\circ} \mathrm{C}[1-3]$, are currently a flourishing area of research. The recent explosion in interest of RTILs has, in part, been due to the designer nature of ions in RTILs [1-3]. Being able to synthesize ions with different chemical constituents and mixing ions in whatever desired combination has delivered liquids with physical properties tuned for niche applications $[4,5]$. Some desirable physical properties of recently developed RTILs include large electrochemical windows, air- and moisture-stability, vanishingly small vapour pressures and resists decomposition up to relatively high temperatures (for detailed reviews see Refs. 1-3). As a result, applications of RTILs are numerous, some of which are as solvents for synthesis and catalysis, lubricants, and electrolytes for fuel cells, batteries, electroactuators and supercapacitors [1-9].

In a capacitor, if the capacitance, $C$, does not depend on voltage, the energy stored, $E=C V^{2} / 2$, goes with the square of the applied voltage, $V$ $[9] .{ }^{2}$ Increasing the voltage window from $1 \mathrm{~V}$ to $3 \mathrm{~V}$, for example, results in almost an order of magnitude increase in the energy stored [9]. Electrolytes in aqueous solvents are typically limited to the former voltage window due to primarily the onset of water electrolysis [3]. RTILs, however, are on average electrochemically more stable in a broader voltage window $[2,3]$. For this reason RTILs have been intensively investigated for application in supercapacitors $[3,9-11]$.

To understand the capacitive abilities of RTILs one must study the electrical double layer (EDL). Theories that describe the EDL of RTILs have provided insight into these interfacial structures and their capacitive performance $[3,7,12-17]$. One relatively recent development, that triggered a flow of work, was to include the finite volume of ions in the mean-field latticegas model [12], which predicted camel or bell shaped differential capacitance curves, instead of the Gouy-Chapman U-shape. Introducing the excluded

\footnotetext{
${ }^{2}$ Differential capacitance curves of electrolytes generally vary with voltage. The integral must be taken with the full voltage dependent differential capacitance if the actual energy stored is to be determined [9].
} 
volume of ions for RTILs was essential since they are ultra-concentrated [12] and can operate at voltages when such effects would be particularly important $[2,3]$. Molecular dynamic simulations [18] following Ref. 12 found that the charge density in EDL oscillate in space [18]. To address charge density oscillations and layering, strong correlations between ions must be accounted for. One of the first theories that reproduced the overscreening EDL structure of RTILs was the Bazant-Storey-Kornyshev theory [7]. That theory [7], however, cannot fully reproduce the charge density profile of atomistic molecular dynamics simulations [19-24] and force-balance measurements [25-29], as the resulting oscillating profiles in that theory [7] decay with the distance from the electrode very rapidly, exhibiting just one or two oscillations [7]. Development of theories that better describe the EDL has attracted much attention [13-17].

One aspect of the EDL in RTILs that has been studied numerous times with experimental [30-37] and computational/theoretical [19-24, 38-41] methods is the temperature dependence. No coherent view has been reached on the dependence and its origin, however; the topic remains to be controversial. Within the computational/theoretical studies [19-24, 38-41], where a variety of RTILs on different electrodes have been investigated, both a positive and negative dependence of linear response capacitance on temperature, and transitions in shapes of differential capacitance curves, have been reported. The former, so-called anomalous temperature dependence of linear response capacitance, has attracted much attention of theorists [42-48]. Previous comparisons between theory and computational/experimental results were typically achieved near the potential of zero charge (PZC). In our present paper, a mean-field theory is presented that includes a route for reductions in the concentration of 'free' ions, similar to the work of Boda et al. [42], that can make predictions for the whole voltage domain.

Refs. 25 and 26 suggested to interpret RTILs as dilute electrolytes [49, 50], assuming that most of the ions are ion paired. Theoretical [51-54], computational [52, 55-59] and experimental $[26,27,60]$ papers have found that the extent of ion pairing in RTILs is not to the extent that was suggested in Refs. 25 and 26 (a detailed review of this work can be found in Refs. 61 and 62). It is almost widely accepted now that the extent of ion pairing suggested in that highly intriguing work [25] cannot be literally taken for RTILs, owing to a number of other physical properties not agreeing with that conclusion $[3,53,56,63]$. In fact, as summarised in Ref. 61, there is a large body of evidence that indicate there are only a small number of Bjerrum 
pairs in bulk RTILs, and that the ion pairing concept is too simplistic for highly concentrated systems [62].

Confined RTILs, on the other hand, are a different story [64-66]. It has been argued that RTILs undergo phase transitions when under nanoconfinement [64-66]. The EDL of RTILs can be studied in confined geometries, but we do not do so here. Instead, we study an atomically smooth electrode; the behaviour of RTILs at which might be expected to reside somewhere between confined RTIL and bulk, offering the possibility of some interesting physics.

This paper comprises of two main sections. The first one focuses on development of the mean-field theory of the EDL in concentrated electrolytes $[12,67]$, and its predictions. Those are then tested, in the second main section, using molecular dynamics simulations of a typical RTIL. There are then two supplementing appendices. The first, namely Appendix A, gives details of the performed simulations. The second, Appendix B, contains additional results that complement the discussion of the main text.

\section{Theory}

On the theory of the EDL in ionic liquids early work [12], it was assumed that all ions in RTILs could participate in screening of electrode charge. The temperature dependence of capacitance only appeared through 'thermal voltage' (given by $k_{B} T / e$, equal to $25.6 \mathrm{mV}$ at room temperature, which sets the scale for electrostatic potential, where $k_{B}, T$ and $e$ are Boltzmann's constant, temperature and elementary charge, respectively), Debye length and a reduction in density from thermal expansion, if any at all [3, 12]. Therefore, a suppression and widening of differential capacitance curves was predicted [12]. ${ }^{3}$ As those formulae overestimated capacitance at PZC, Stern layers were introduced $[3,18,88,89]$. The wings of capacitance curves have no explicit temperature dependence, and Stern layers are expected to only have a minor one. Therefore, the Kornyshev-Stern theory can not explain both the positive and negative temperature dependences, and transitions in

\footnotetext{
${ }^{3}$ Thermal expansion would act to reduce the concentration of ions. This predicts that capacitance curves go in the direction of bell to camel shape. Observing such a transition would require extreme temperatures, however, beyond the temperature range of applications.
} 
shapes of differential capacitance that have been reported in the literature [19-24, 30-40].

Evidently, if the reported temperature dependences of differential capacitance curves are to be rationalised, an extension to that theory [12], beyond the mean-field of fully dissociated ions, is required. Below the lattice-gas mean-field theory is developed, which does not a priori assume the value for the fraction of ions that can participate in screening of electrode charge $[53,54,68]$. The modification is simple and can be readily interpreted. However, it does not yield much predictive power over the fraction of 'free' charge carriers, as it is determined by a free parameter that controls the balance between the 'spectating' and 'participating' ions. It thus gives an option for the conversion between the two states, in a manner similar to the theory of intrinsic semiconductors [3], which gives rise to additional temperature dependent effects. Rather, the theory contributes to the interpretation of molecular dynamics simulations to provide further insight into the EDL properties.

\subsection{Basic Equations and Concepts}

Consider a model with participating ions and spectating ions distributed over a ?lattice? [53]. The former comprises of cations and anions, of which there are $N_{+}$and $N_{-}$, respectively, that posess net charge [53]. Electroneutrality in the bulk requires that $N_{+}=N_{-}=\bar{N} / 2$. While spectating ions, considered to belong to ion pairs and neutral aggregates [61], bear no net charge. They appear in the theory as $N_{s}$ neutral lattice sites that behave in a similar way to voids [12], but their number is not fixed (note spectating ions still occupy a finite volume). Each of the $N$ lattice sites is occupied by participating or spectating ions, $N=N_{+}+N_{-}+N_{s}$.

Voids [12] have been neglected here. The reason for doing so is two fold. Firstly, there have been numerous studies into the fraction of free volume (free volume per unit volume) in RTILs [69-84]. These have generally reported that the fraction of free volume is in the range of 0.05-0.2 [69-78]. For free volume to be considered as a void it must be treated on the same footing as ions; that being, it has a similar volume. The proportion of free volume that can be considered as voids has been found to be vanishingly small [7984]. Further justification neglecting voids has been presented in the Free Volume section of Appendix B.2. Although neglecting them may still be considered as model approximation which greatly simplifies the theory. As a rigorous, quantitative theory is not sought after here, but a heuristic, simple one, neglecting this small contribution is prudent. 
The free energy that describes this two state extension can be approximated as

$$
\begin{gathered}
F=e \Phi\left(N_{+}-N_{-}\right)+\left(U_{p}-T S_{p}\right)\left(N_{+}+N_{-}\right)+\left(U_{s}-T S_{s}\right)\left(N-N_{+}-N_{-}\right) \\
+\frac{z A_{+}}{2} \frac{N_{+}^{2}}{N}+\frac{z A_{-}}{2} \frac{N_{-}^{2}}{N}+z B \frac{N_{+} N_{-}}{N}-k_{B} T \ln \left\{\frac{N !}{\left(N_{+}\right) !\left(N_{-}\right) !\left(N-N_{+}-N_{-}\right) !}\right\} .
\end{gathered}
$$

The contribution to the energy from the interactions of participating ions, which appear through the charge density $e\left(N_{+}-N_{-}\right)$, with the electrostatic field, $\Phi$, is given by the first term. Participating and spectating ions have an intrinsic free energy in this 'two state theory', denoted with subscript $p$ in the second term and $s$ in the third term, respectively. The formalism presented here for determining the concentration of participating ions is very similar to that of defects in crystals [85]. Regular solution short-range correlations [6, $53,86]$ between participating ions have been included to reproduce simulation data without a Stern layer [86]. No correlations between spectating ions are considered, for simplicity. Short-range correlations constants between cations, between anions, and between cations and anions are given by $A_{+}$, $A_{-}$and $B$, respectively, with a constant coordination number, $z$, assumed for each ion. Lattice entropy is described by the last term $[3,12,53,86]$.

In concentrated electrolytes, correlations between ions result in decaying oscillations in charge density [14-16, 25-29]. The utilised mean-field theory can not reproduce those oscillations, but it has gone beyond the mean-field theory of fully dissociated ions. Decaying oscillations in charge density can be decomposed into a monotonic decay and an oscillating component [53]. The monotonic decay is captured with mean-field theories [12, 67]. While the oscillating component could be interpreted as ion pair orientation [87]. Hence, from introducing spectating ions, which represent ion pairs and neutral aggregates, we can include some effects of strongly correlated systems $[13-16,87]$.

\subsection{Participating Ion Fraction}

As mentioned, the extension to the mean-field theory that introduces the two states for ions, reminiscent of semiconductor theory $[3,53]$. The relative energy of these two states decides, in part, the extent of the 'free' charges 
in the system. As spectator ions should be related to ion pairs and neutral aggregates, it is intuitive to expect that the energy level of spectating ions is lower than that of participating ions. There is thus a 'band gap' between spectating ions and participating ions for thermal activation, in a similar way that there is a band gap between the conduction and valence band in semiconductors. Much like how electrons are thermally activated from the valence band to conduction band, ions should be thermally activated from the spectating state to participating state. With increasing temperature, one would expect that complete dissociation of ions occurs. This limiting behaviour is captured through the intrinsic entropy term of the free energy. From mean-field theories of the EDL it is well known that there needs to be a change in the concentration of 'free' charge carriers if there is to be a transition in the shape of the differential capacitance curve $[3,12,53,86]$. Thus, previewing the results, the temperature in this theory may regulate the transition from camel to bell shape.

Since a two state theory has been introduced in the place of voids, the interpretation of $\gamma=\bar{N} / N^{4}$ has been altered, but the essence of the parameter remains. Previously $\gamma$, referred to as compacity, described the extent to which charge could amplify in the EDL, owing to the presence of voids. Now referred to as the participating ion fraction, $\gamma$ still measures the extent to which participating ions can accumulate, but from replacing spectating ions rather than voids. The participating ion fraction now depends on a number of factors, such as the 'band gap' $\left(\chi_{0}\right)$ and temperature.

Stirling's approximation can be taken to Eq. (1), and the resulting equation can be converted to a dimensionless, intensive free energy $(f=$ $\left.F / k_{B} T N\right)$. Minimising $f$ with respect to $\gamma$ obtains an equation on participating ion fraction in the bulk

$$
\frac{\partial f}{\partial \gamma}=\chi_{1} \gamma / 2+\chi_{0}+\ln \left\{\frac{\gamma / 2}{1-\gamma}\right\}=0
$$

where

$$
\chi_{1}=\frac{a_{+}+a_{-}}{2}+b, \quad \chi_{0}=\left(u_{p}-T s_{p}\right)-\left(u_{s}-T s_{s}\right)=\Delta u-T \Delta s .
$$

\footnotetext{
${ }^{4}$ Also seen as $\gamma=\bar{c} / c_{\max }$, where $\bar{c}$ and $c_{\max }$ are bulk and maximum concentration of participating ions, respectively.
} 
Short-range correlation constants and intrinsic free energy terms have been rescaled to thermal energy, $k_{B} T$, denoted by lower case letters.

To simplify the analysis, we neglect the first term on the r.h.s. of Eq. (2) assuming $\chi_{1}$ is a few times smaller than $\chi_{0}$. One can then solve Eq. (2) for the participating ion fraction

$$
\gamma=\frac{1}{1+\frac{1}{2} \exp \left\{\chi_{0}\right\}}
$$

to obtain an equation that resembles a Fermi function. When $\chi_{0}=0$, Eq. (2) gives $2 / 3$ for the participating ion fraction, which is consistent with an estimate for bulk RTIL [51], both of which are temperature independent. However, one should be careful with this limit, because in this case the $\chi_{1}$ term in Eq. (2), of course, can not be neglected. ${ }^{5}$

\subsection{Capacitance}

For the symmetric case, $a_{+}=a_{-}=a$, equations from Ref. [86] can be utilised for differential capacitance. ${ }^{6}$ There [86] the rescaled set of equations for Debye length and potential was found from an interpolation result that originated from a linear response analysis. The resulting analytical expression for the differential capacitance

$$
C=\tilde{C}_{0} \frac{\cosh \left\{\alpha u_{0} / 2\right\}}{1+2 \gamma \sinh ^{2}\left\{\alpha u_{0} / 2\right\}} \cdot \sqrt{\frac{2 \gamma \sinh ^{2}\left\{\alpha u_{0} / 2\right\}}{\ln \left\{1+2 \gamma \sinh ^{2}\left\{\alpha u_{0} / 2\right\}\right\}}},
$$

with

\footnotetext{
${ }^{5}$ An iterative estimate will give in this case, $\gamma \sim 1 /\left(1+\exp \left\{\chi_{1} / 3\right\} / 2\right)<2 / 3$, in which the first term on the r.h.s. of Eq. (2) is replaced by $\chi_{1} / 3$.

${ }^{6}$ It should be noted that the only contribution to the differential capacitance discussed here is from the EDL of the RTIL. No contribution has been considered from the electrodes space charge. This is a good approximation when the electrodes are metallic. For lowdimensional carbon electrodes, such as graphene [90-92] and graphite [93], only considering the EDL of the RTIL is not adequate. At linear response, the differential capacitance contribution from the electrode dominates, and therefore, must be included if one is to describe the full capacitance-voltage dependence [93-95].
} 


$$
\alpha=\frac{1}{1+\frac{\gamma}{2}(a-b)}
$$

was able to reproduce typical values of linear response capacitance and the dependence of differential capacitance on voltage. Here $\tilde{C}_{0}$ is rescaled Debye capacitance, given by $\epsilon \epsilon_{0} \tilde{\kappa}$, in SI units, where $\epsilon_{0}$ and $\epsilon$ are permittivity of free space and relative permittivity, respectively; $u_{0}$ is dimensionless potential drop across the double layer, seen as $\mathrm{e}_{0} / \mathrm{k}_{B} \mathrm{~T}[12,86]$. The rescaled inverse Debye length is $\tilde{\kappa}=\sqrt{\alpha} \kappa$. Here, $\kappa=\sqrt{q^{2} \bar{c} / \epsilon_{0} \epsilon k_{B} T}$ is the inverse Debye length where $q$ is the absolute value of charges of ions and $\bar{c}$ is the bulk concentration of the total number of ions in the RTIL [12].

Temperature dependence of Eq. (5) arises in a number of places. Already mentioned, 'thermal voltage', acts against charge accumulation with increasing temperature, broadening the curve as temperature increases [3]. In the potential rescaling factor, $\alpha$, temperature also rescales short-range correlations, reducing their 'strength' as temperature increases. Both of these factors, thermal voltage and $\alpha$, act against each other to result in an almost temperature independent factor which multiplies electrostatic potential. The temperature also appears in the Debye length, increasing its length as temperature becomes higher. There is an additional, 'hidden' temperature dependence of $\gamma$, cf. Eq. (4), as the dimensionless parameter $\chi_{0}$ is scaled to thermal energy, which is the new aspect of the mean-field theory.

This version predicts, if there is a preference for spectating ions at low temperatures and participating at high temperatures, a transition in the capacitance-voltage dependence with temperature. Fig. 1 demonstrates such a transition from a camel to bell shape with increasing temperature. The explanation for this is as follows: with increasing temperature there is dissociation of ion pairs and neutral aggregates that cause the 'free' ion fraction to increase.

Substituting Eq. (4) into $\tilde{C}_{0}$ obtains an equation that describes the temperature dependence of the linear response capacitance

$$
\tilde{C}_{0}=\sqrt{\frac{\epsilon_{0} \epsilon q^{2} c_{\max }}{k_{B} T} \frac{2}{2+(a-b)+\exp \left\{\chi_{0}\right\}}} ;
$$

which retains the same qualitative features as the anomalous temperature dependence obtained in Refs. [42-48], as seen in Fig. 2. At low temperatures, 


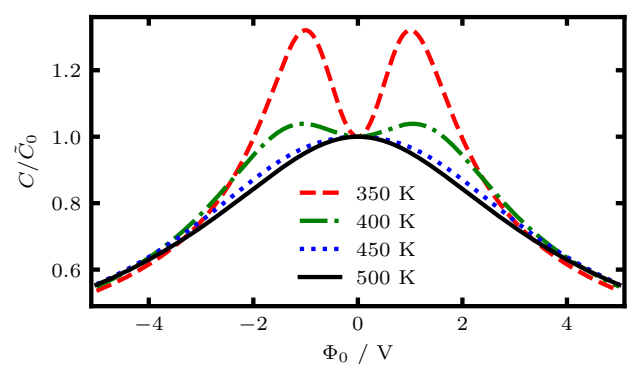

Figure 1: Differential capacitance curves transition from camel to bell shape with increasing temperature, as predicted from the modified mean-field theory. Differential capacitance scaled to Debye capacitance as a function of voltage drop across the EDL for the indicated temperatures. Here $\Delta U=2300 k_{B} K, \Delta S=-4 k_{B}$ and $z(A-B)$ $=5 \times 10^{5} k_{B} K$. (The strength of the differences in the absolute values of capacitance will be dampened by $\tilde{C}_{0}$, cf. Figs. 2 and 3 )

it would be expected that the values of $\gamma$ are small. With increasing temperature spectating ions are thermally excited to participating ions, and so $\gamma$ increases. This effect dominates at low temperatures and results in a positive temperature dependence of linear response capacitance. There comes a temperature when the increase in capacitance from this source is cancelled from temperature appearing in the denominator of Eq. (7) through the value of $\chi_{0}$. Therefore, a stationary point in capacitance is reached. For larger temperatures, the temperature dependence of linear response capacitance is predominantly governed by the temperature dependent factor $\chi_{0}$ in the denominator of Eq. (7).

\section{Simulation}

Here a classical molecular dynamics (MD) simulation of 1-ethyl-3-methylimidazolium bis(trifluromethanesulfonyl)imide ([Emim] $\left[\mathrm{Tf}_{2} \mathrm{~N}\right]$ ) confined between atomically smooth electrodes at different temperatures was performed (all of the details of which can be found in Appendix A). In this section the differential capacitance curves of $[\mathrm{Emim}]\left[\mathrm{Tf}_{2} \mathrm{~N}\right]$ at different temperatures are presented, and the observed transitions described in comparison to what is expected from the presented mean-field theory. The origin of those trends are then discussed in some detail. 


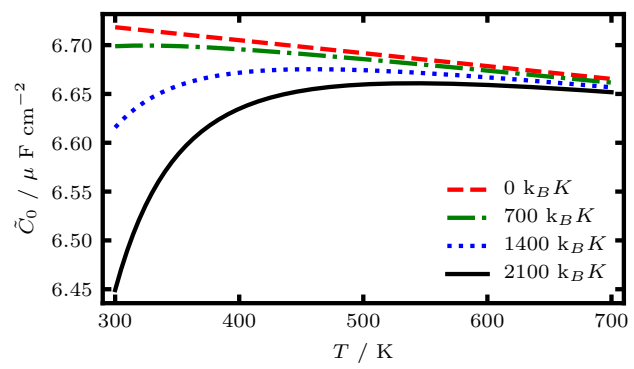

Figure 2: Linear response capacitance can exhibit both positive and negative temperature dependences. Rescaled linear response capacitance as a function of temperature for the indicated changes in intrinsic internal energy, $\Delta U$. Here $c_{\max }=6 \mathrm{M}, \epsilon$ $=3, \Delta S=-4 k_{B}$, and $z(A-B)=5 \times 10^{5} k_{B} K$.

\subsection{Differential Capacitance}

Numerous dependences of differential capacitance curves on temperature have been reported for RTILs [19-24, 30-41]. The calculated differential capacitance from our MD simulations, shown in Fig. 3, where a theoretical plot has been included for comparison, is similar to those reported in Refs. 20 and 24. Generally, differential capacitance is found to be temperature dependent at small voltages, but temperature independent at large voltages. Indeed the latter is well known $[3,12]$. According to the charge conservation law $[3,12]$, differential capacitance does not explicitly depend on temperature and goes with the inverse square root of potential drop across the double layer (a comparison of MD simulations and theory for large voltages can be seen in Appendix B.1). A positive temperature dependence was found for linear response capacitance (see Appendix B.1). At intermediate voltages the dependence of differential capacitance on voltage changes from increasing to decreasing with increasing temperature; simply referred to as a transition from a camel to bell shape. As noted in the theory section, changes in the shapes of differential capacitance curves for a given electrolyte generally occur through variation of the charge carrier concentration. Therefore, the differential capacitance curve suggests that with increasing temperature the concentration of charges increases, which can occur through dissociation of ion pairs and neutral aggregates. In Ref. 41 a similar conclusion was reached, where a 'frozen' structure was reported to 'melt' with increasing temperature.

Comparing the theoretical curve to the MD one finds good agreement in 

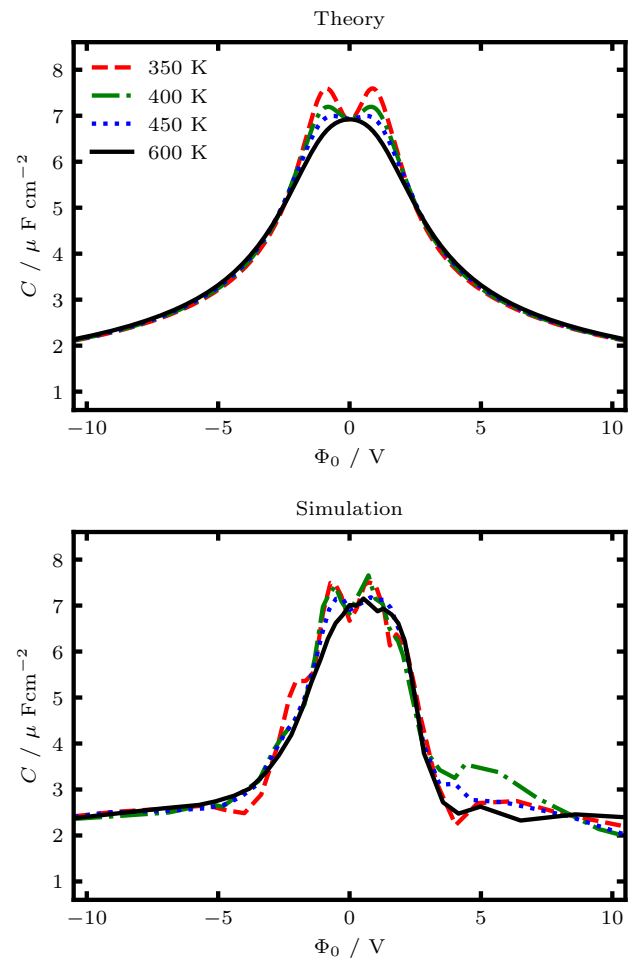

Figure 3: Differential capacitance transitions from a camel to bell shape with increasing temperature. (top) Theoretical differential capacitance as a function of electrode potential, at the indicated temperatures, for $\epsilon, q$ and $c_{\max }$ at 1.6, 0.8e and 7.82 M [27], while $\Delta u, \Delta s$ and $a-b$ took on $7 / 3,0 \mathrm{~K}^{-1}$, and 100 at $300 \mathrm{~K}$. (bottom) Differential capacitance as a function of electrode potential, calculated from MD simulations, for the indicated temperatures. For details of simulations see Appendix A.

the qualitative trends and quantitative values. This fact is remarkable since this version of the mean-field theory does not describe layering of ions at the interface and the corresponding overscreening effect [7], neither involves any molecular details of the liquid. Can the reported trends be expected to apply to other RTILs? The trends shown here are in agreement with those reported in the literature for a set of liquids [20, 24]. There were also other trends reported [19, 21-23, 38-41] which can be conditioned with the presented theory, but we have not investigated those cases here.

There are a few elements of the presented mean-field theory that enabled 
good agreement with the MD simulation. Introducing short-range correlations permits a simple, analytical formula for differential capacitance that can reproduce linear response capacitance and the dependence of differential capacitance on voltage [86]. The new element of the theory, namely the introduction of two states for ions, is able to capture the transition in shape of capacitance-voltage curves with temperature [53]. This transition corresponds to thermal activation of spectating ions to participating ions, which is consistent with dissociation of ion pairs and neutral aggregates. As already mentioned, the two state theory is a simplified approach to rationalise correlations beyond the mean-field of fully dissociated ions.

\subsubsection{Origin of Temperature Dependence}

At small and intermediate voltages the differential capacitance curve is temperature dependent. There should, then, be temperature dependent charge density distributions that result in the temperature dependence of differential capacitance. Fig. 4 displays the change in charge density $\left(\rho-\rho_{0}\right.$, where $\rho$ and $\rho_{0}$ are charge density at the indicated surface charge and charge density at no applied surface charge, respectively). With increasing surface charge, close to PZC, the amplitudes of oscillations in charge density become more pronounced. This fact is generally accepted and currently an active area of research for EDL theories [3, 13-17]. Comparing the same electrode, for a given voltage, at different temperatures shows that the change in charge density distribution is suppressed and smoothened with increasing temperature $[22,41]$.

Differential capacitance is defined as the derivative of surface charge density with respect to potential drop across the EDL [3]. Therefore, smaller gradients of surface charge with respect to voltage corresponds to a lower differential capacitance. The reduction of the change in charge density with increasing temperature is consistent with the reported change in differential capacitance curves, then. The presented mean-field theory states that ion pairs and neutral aggregates should dissociate with increasing temperature. This results in the observed transition in shape of differential capacitance curves.

At large voltages the differential capacitance becomes temperature independent $[3,12]$. This fact, which is a model-independent feature, should also be seen in the charge density distributions. Fig. 5 compares the temperature dependence of charge density at PZC and at large voltages. For the charge density plots at PZC there is clearly temperature dependence, where corre- 

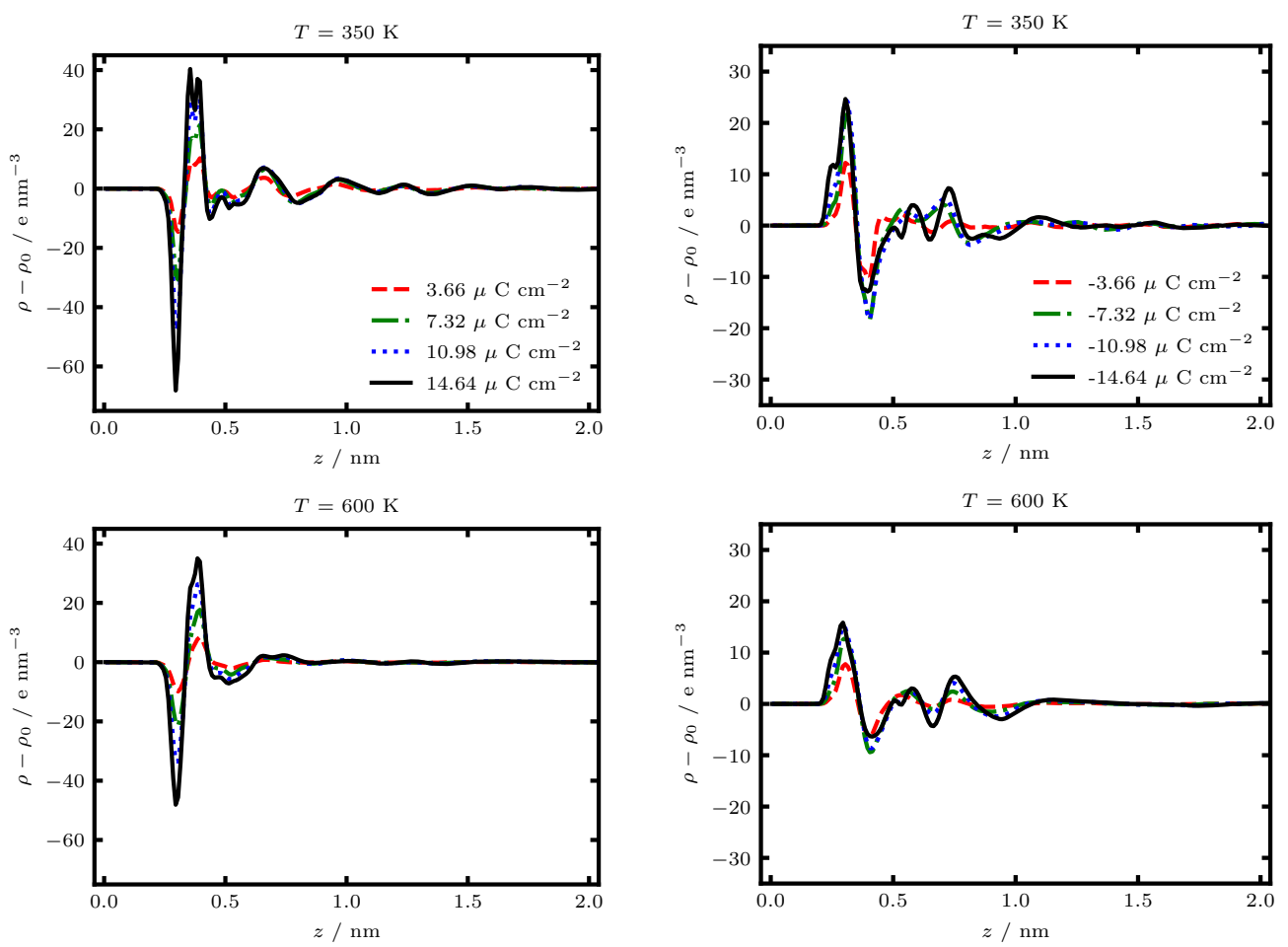

Figure 4: Temperature suppresses the response of the change in charge density with respect to applied voltage. Change in charge density, $\rho-\rho_{0}$, as a function of displacement from the electrode for the indicated surfaces charges (anode - left; cathode right) and temperature [41].

lated ion pairs and neutral aggregates are broken up by temperature [41]. In contrast, large voltages have effectively temperature independent charge density distributions. Both of these observations are in agreement with the theory.

To summarise thus far, temperature dependent differential capacitance at small and intermediate voltages has revealed a transition from camel to bell shape with increasing temperature [20, 24, 41]; this temperature dependence was shown to be consistent with the charge density distributions, where ordered layers (see Appendix B.2 for further evidence for a glassy state at the interface) of ions at the interface are disrupted with increasing temperature 

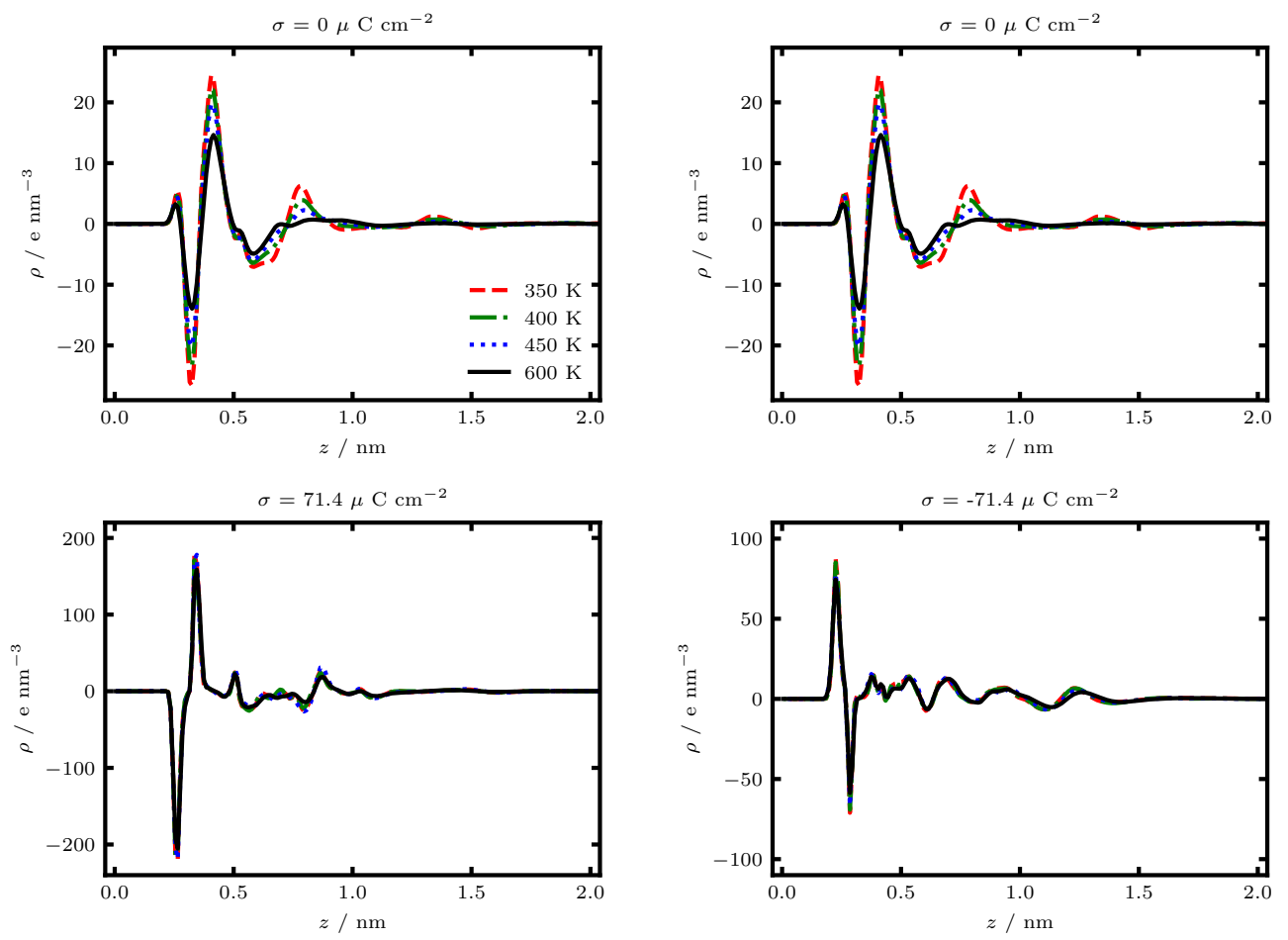

Figure 5: Temperature dependent charge density distribution at PZC, and temperature independent charge density distribution at large applied surface charges. Charge density, determined from partial charges on atoms, as a function of displacement from the electrode for the indicated temperatures. PZC cases shown in the top Fig. and large applied voltage in the bottom Fig. (anode - left; cathode - right).

[41]; the presented theory has demonstrated that the observed transitions originate from thermal activation of spectating ions to participating ions, i.e. changes in the concentration of charge carriers with temperature.

Before the intriguing report of the Israelachvili group [25, 26], it was generally accepted that RTILs were similar to molten salts [3, 12], in the sense that most of the ions were not paired. Since then, there has been a great deal of discussion regarding those measurements and if RTILs are, in fact, 'dilute electrolytes' [3, 49-59, 62, 63].

The simulations performed here indicate that ion pairs can form at the interface of electrodes and result in temperature dependent differential ca- 
pacitance curves. At low temperatures, these orientated ion pairs can be seen to extend over $1 \mathrm{~nm}$ from the electrode. Higher temperatures dissociate ion pairs, which reduces the amplitude of decaying oscillations and electroneutrality is reached in a shorter distance from the electrode. What, then, is the extent of ion pairing at the interface?

It is possible to fit differential capacitance curves to find a value of $\gamma \cdot{ }^{7}$ Upon doing so, values of $\gamma$ are $0.21,0.20,0.26$ and 0.40 in ascending order of the studied temperatures. Hence, there appears to be substantial reductions in the concentration of 'free' charge, but not to the extent reported by the Israelachvili group $[25,26]$.

\section{Conclusion}

Theoretically, we applied the concepts of earlier work, capable for testing the full voltage window. More specifically, 'ion pairs' and 'neutral aggregates' were incorporated, in terms of net charge - not structure, into the theory; in contrast to previous mean-field theories for the electrical double layer in ionic liquids, voids are neglected, motivated by experiments and the prospect of a simpler theory. An anomalous temperature dependence of linear response capacitance, similar to the work of Boda et al. [42], also resided in the presented mean-field theory. The new observation of the developed meanfield theory was that differential capacitance curves transform form a camel to bell shape with increasing temperature, rationalised as thermal activation of spectating ions to participating ions.

Molecular dynamics simulations of 1-ethyl-3-methylimidazolium bis(trifluromethanesulfonyl)imide, performed confined between atomically smooth electrodes, tested the predictions of the developed theory. Simulated differential capacitance curves showed signatures of a transition from camel to bell shape with increasing temperature, and an increasing linear response capacitance, congruent with the theoretical prediction. The origin of the temperature dependence was further investigated in the charge density distributions. A disordering of regular ionic layers at the electrode, as earlier observed [41], is consistent with dismantling of 'ion pairs' and 'neutral aggregates'. Therefore, the main conclusion of the developed theory, despite its simplicity, agrees well with the performed molecular dynamics simulations.

\footnotetext{
${ }^{7}$ See Appendix B.1 for these fitted curves.
} 


\section{Acknowledgements}

Special thanks are to Prof. M. Fedorov for many discussions of the possible temperature effects on the properties of electrical double layer in ionic liquids that preceded this work. Discussions with Prof. M. Bazant, Prof. F. Bresme and O. Robotham are greatly appreciated. GF and MC acknowledge the funding support from the National Natural Science Foundation of China (51406060) and Shenzhen Basic Research Project (JCYJ20170307171511292); ZG was supported through a studentship in the Centre for Doctoral Training on Theory and Simulation of Materials at Imperial College London funded by the EPSRC (EP/L015579/1) and from the Thomas Young Centre under grant number TYC-101.

\section{Appendices}

\section{A. Simulation Details}

Classical molecular dynamics (MD) simulations were utilised to calculate the microstructure and differential capacitance of a RTILs EDL. As shown in Fig. 6, the modelled channel system consists of a slab of RTIL, namely 1ethyl-3-methylimidazolium bis(trifluromethanesulfonyl)imide (Emim] $\left[\mathrm{Tf}_{2} \mathrm{~N}\right]$ ), enclosed between two electrodes, each of which consists of three graphene layers. The simulation box was $4.26 \times 4.92 \times 30 \mathrm{~nm}^{3}$, with a distance of $8 \mathrm{~nm}$ between the two electrodes, which is sufficient to ensure that the RTIL in the central region of channel is in a bulk-like state. The number of cations and anions was adjusted until the density in the channel center matched that of the bulk RTIL, within a deviation of $1.0 \%$ for various mass densities at different temperatures.

The simulations were performed in NVT ensemble with the MD package Gromacs 4.6 [96]. The velocity Verlet algorithm [97] was employed to integrate Newton's equation with a time step of 2 fs. Temperature was maintained by using Nosé-Hoover coupling [98] with a time constant of 0.5 ps.

The force field of [Emim] $\left[\mathrm{Tf}_{2} \mathrm{~N}\right]$ was taken from Yong et al.[99] Partial charges of cations and anions were taken to be \pm 0.8 e as an effective way to

account for electronic polarizability of ions in RTILs [100]. The force field of electrode was taken from the work of Cornell et al. [101]. 


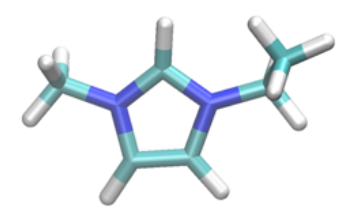

$[\text { Emim }]^{+}$

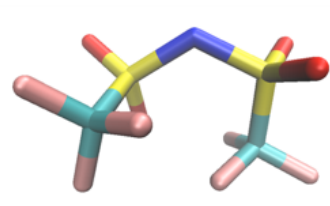

$\left[\mathrm{Tf}_{2} \mathrm{~N}\right]^{-}$

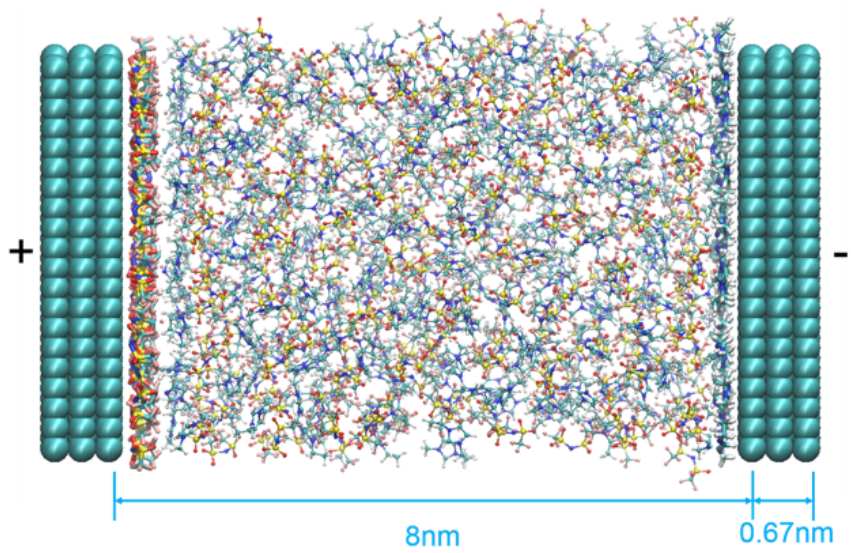

Figure 6: Schematics of a MD simulation cell for $[\operatorname{Emim}]\left[\mathrm{Tf}_{2} \mathrm{~N}\right] /$ graphene supercapacitors. The RTIL was confined by two planar graphene electrodes modelled by three graphene layers.

Coulombic interactions were computed using slab-PME to ensure accuracy of the electrostatic force in two-dimensional periodic geometry adopted here [102]. Specifically, the dimension vertical to the electrode of the simulation box was set to be 3.75 times than the electrode separation, which is sufficient to ensure the accuracy of the electrostatic force calculation compared to that of the two-dimensional Ewald summation method [103]. To compute the electrostatic interactions in reciprocal space, an FFT grid spacing of $0.12 \mathrm{~nm}$ and cubic interpolation was used. A Coulombic cutoff of 1.2 $\mathrm{nm}$ was adopted to calculate the electrostatic interaction in real space. Nonelectrostatic interactions were computed by direct summation with a cutoff length of $1.2 \mathrm{~nm}$.

\section{A.1. Differential Capacitance Calculation Method}

For each simulation, the MD was run 20 ns to reach the equilibrium state, and another $10 \mathrm{~ns}$ for trajectories for further analysis. Using the obtained 
MD trajectories, for each value of surface charge density, given by $\sigma$, the charge density distributions normal to the electrode were calculated using bins with a width of $0.01 \mathrm{~nm}$.

The second integral of Poisson's equation

$$
\frac{d^{2} \Phi(z)}{d z^{2}}=-\frac{\rho}{\epsilon_{0}}
$$

as seen in SI units, affords the electrical potential distribution

$$
\Phi(z)=-\frac{\sigma}{\epsilon_{0}}-\frac{1}{\epsilon_{0}} \int_{0}^{z}\left(z-z^{\prime}\right) \rho\left(z^{\prime}\right) d z^{\prime} .
$$

where $\Phi(z), \rho$ are the potential distribution and charge densities along $z$ direction, respectively. From this, one can obtain the potential drop across the EDL, $\Phi_{0}$. To compute differential capacitance, a piecewise fourth-order polynomial was fitted [104] and the derivative taken to determine differential capacitance $\left(C=d \sigma / d \Phi_{0}\right)$. Note that only the contribution from the EDL has been considered in the calculation for differential capacitance, so a comparison can be made directly to the presented mean-field theory.

\section{A.2. Free Volume Calculation Method}

The free volume fraction, $v_{f}$, (free volume per unit volume) is commonly reported in experiments and MD simulations [69-83, 105]. Here, we investigate two kinds of free volume: that without a probe and with an argon probe (radius of $0.188 \mathrm{~nm}$ ), which is a test for a void (volume on a similar footing to ions). The free space without a probe was simply determined from the number of ions and their respective volumes, determined from the atoms van der Waals radii [106]. ${ }^{8}$ The void free space can be determined by inserting a spherical probe in the simulation box. Probes that do not overlap with any atoms in molecules, volumes of which were determined from their van der Waals atomic radii [106], are considered to belong to a free region.

\footnotetext{
${ }^{8}$ The van der Waals radii determined by Bondi [106] were used for the probe and ionic liquids atoms.
} 


\section{B. Supplementary Results}

\section{B.1. Additional Results from Differential Capacitance Analysis}

In Fig. 7 a comparison between the theory (Fig. 3) and simulation for the universal charge conservation law $[3,12]$. Reasonable agreement between the two are observed.

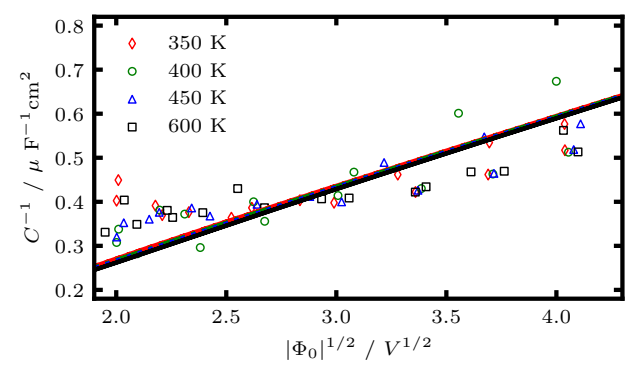

Figure 7: Reasonable agreement between theory and simulation for the charge conservation law. Reciprocal differential capacitance as a function of the square root of the potential drop across the double layer at the indicated temperatures. For the parameters used in the theory see Fig. 3.

Linear response capacitance at different temperatures, for theory and simulation (seen in Fig. 3), is displayed in Fig. 8. A positive temperature dependence of linear response capacitance was observed for the simulations. Reasonable curve fits attain values of linear response that do not depend as strongly on temperature. The observed dependence of linear response on temperature can be conditioned with the presented mean-field theory, but the corresponding differential capacitance curves do not reproduce the simulations well. There my be two reason for it: (i) - the accuracy of simulated values close to $\mathrm{PZC}$ is not high due to pronounced noise; (ii) - inability of the mean-field theory to describe overscreening, particularly important near PZC.

For reference, Fig. 9 displays the fitted differential capacitance curves to attain $\gamma$, and therefore, the reduction in charge carrier concentration. Only $\gamma$ was varied when fitting. All other parameters remained constant and at that indicated in Fig. 3 of the main text. To reiterate, the values of the fitted $\gamma$ were found to be $0.21,0.20,0.26$ and 0.40 in ascending order of the studied temperatures. These values do not correspond to one value of the 


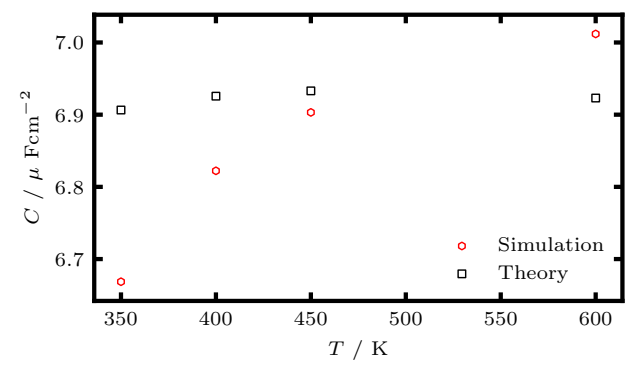

Figure 8: A positive temperature dependence of linear response capacitance is obtained for the studied RTIL. Linear response capacitance as a function of temperature, for theory and simulation. Parameters used in the calculation are the same as those in Fig. 3.

'band gap', $\chi_{0}$, for the RTIL, but different ones since it is non-monotonic. In Fig. 3 of the main text, a curve was displayed that had one value of the band gap, from which the corresponding values of $\gamma$ can be obtained. For the considered band gap, see Fig. 3 for its numerical value, $\gamma$ was found to be $0.21,0.26,0.30$ and 0.38 , in ascending order of the studied temperatures, which is not far from the fully fitted values.

\section{B.2. Free Volume Determination}

There has been a great deal of literature concerned with the free volume in RTILs. It has been reported the that fraction of free volume, $v_{f}$, in bulk RTILs is in the range of 0.05-0.2 [69-83]. The volume of an average void is expected to be smaller than the volume of ions. In fact, the probability of voids with a similar volume to ions is in the range of $10^{-4}-10^{-5}$ [79, 80]. Fig. 10 displays the determined bulk free volume of the studied RTIL from the MD simulations (more details can be found in Appendix A). If we want to compare the free volume estimates obtained without a probe, we must realise that not all of the latter can be utilised for compression/electrostriction. Indeed, even a dense packing of hard spheres will have a volume of the interstitial space, which the system can never get rid of. Thus the data points presented as circles should be multiplied by a factor that could in principle, be as small as $0.63[7]$. Upon doing so, a value of roughly 0.2 is found, which is in good agreement with experiments of RTILs [69-78]. The free volume over a threshold can be determined with the use of a probe, which has a 

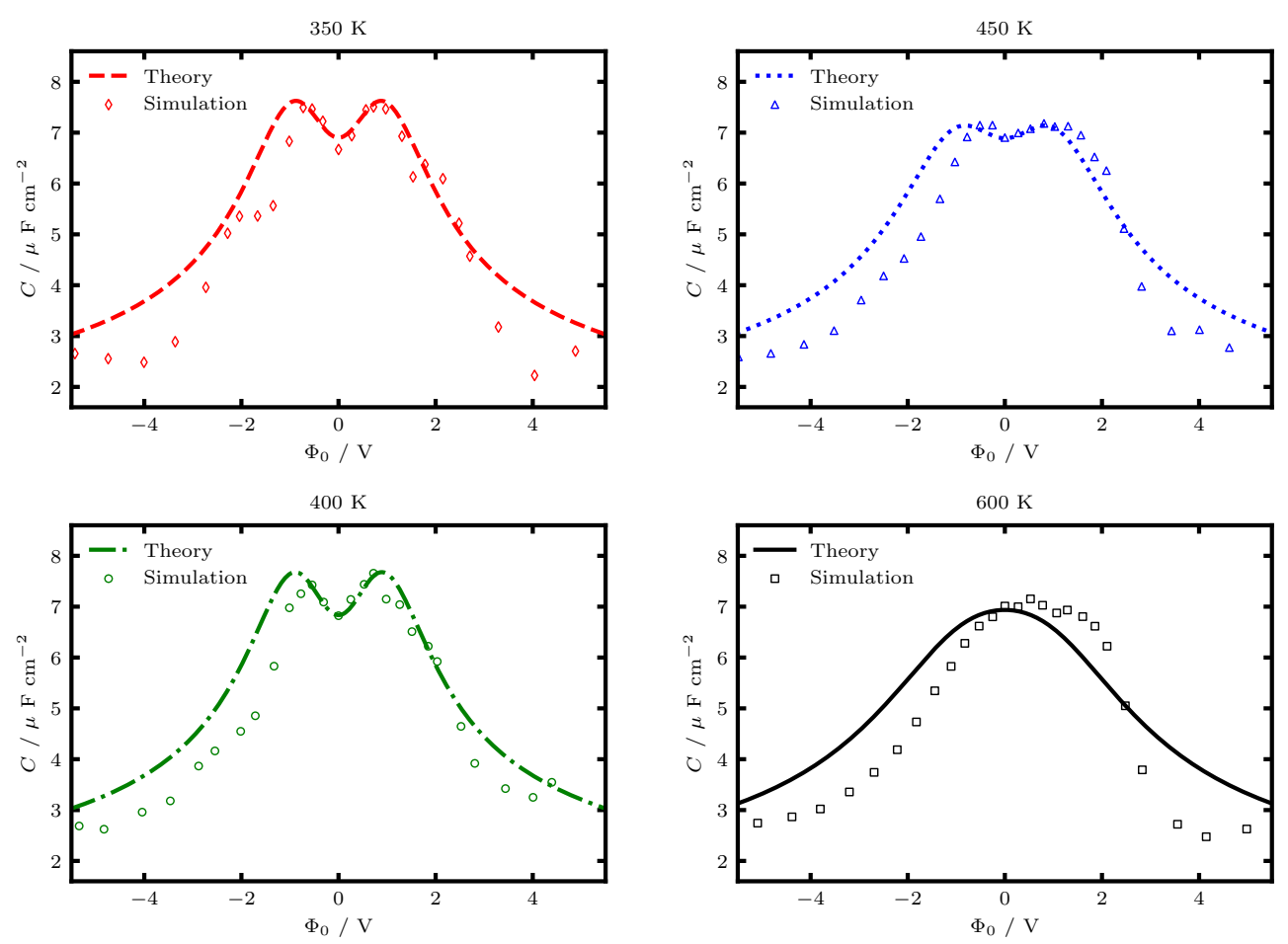

Figure 9: Fitted differential capacitance curves show reasonable agreement over the whole voltage domain with the potential rescaled differential capacitance equation. Differential capacitance as a function of applied voltage for the indicated temperatures. These were the curves used to obtain values of $\gamma$. Values of the fitted $\gamma$ were found to be $0.21,0.20,0.26$ and 0.40 in ascending order of the studied temperatures.

finite volume similar to ions, and a value close to zero is attained (values in ascending order of temperature were $0,0,1 \times 10^{-4}$ and $2 \times 10^{-3}$ ). This is also in good agreement with literature [79, 80], and justifies neglecting voids in the theory.

Comparatively, studies of the free volume in EDLs of RTILs are scarce, and the temperature and surface charge dependence are essentially nonexistent in the literature. Fig. 11 displays the distribution of free volume as a function of displacement from the interface, which shows that the region near the electrodes has a smaller fraction of free volume. Typically, glassy RTILs (a disordered solid phase) have free volume fractions smaller than the 


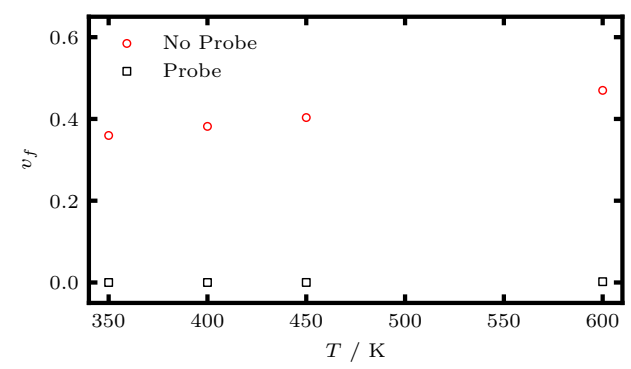

Figure 10: There is essentially no voids in RTILs. Free volume fraction as a function of temperature with a probe and without a probe.

value of liquid RTILs [74, 76-78]. Hence, the free volume is consistent with the observation of oriented ion pairs and neutral aggregates at the electrode interface [41].

The charge density distributions, see Figs. 4-5, showed that the 'frozen' layers at the interface 'melt' with temperature. This can further be seen in the free volume fraction distributions, where the glassy layer near the interface has more free volume with increasing temperature. 

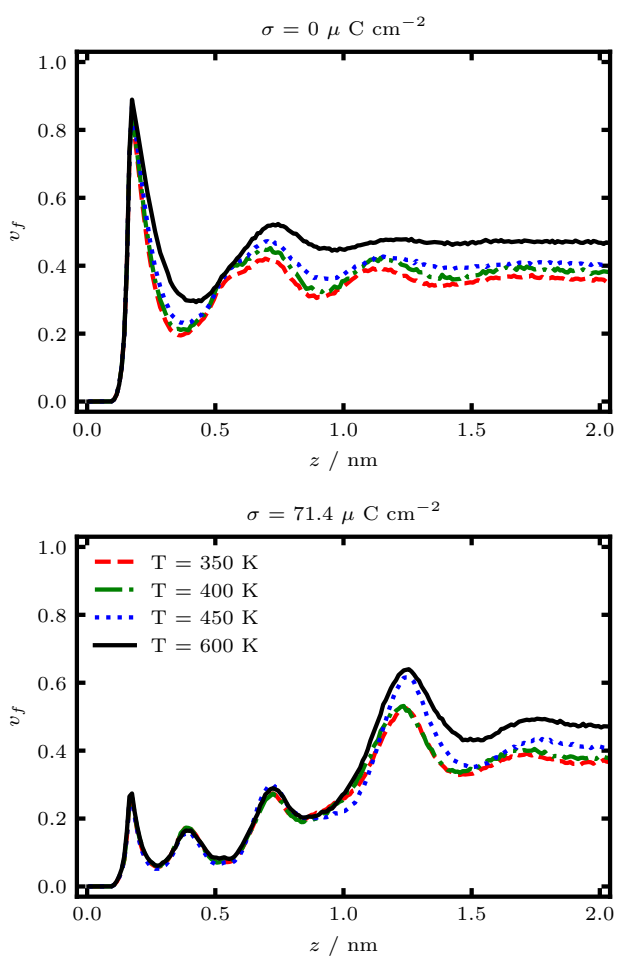

Figure 11: The comparison of the two panels shows that electrostriction diminishes the void space, and this happens, as expected, predominantly within the first layers near the interface. But even at non-polarized electrode the portion of void space is smaller than in the bulk, indicating at the denser packing of RTIL ions near the solid-liquid interface. Local distribution of the free volume near non-polarized (top) and near charged (bottom) electrode.

\section{References}

[1] T. Welton, Room-temperature ionic liquids. solvents for synthesis and catalysis, Chem. Rev. 99 (1999) 2071-2083.

[2] H. Weingärtner, Understanding ionic liquids at the molecular level: Facts, problems, and controversies, Angew. Chem. Int. Ed. 47 (2008) 654-670.

[3] M. V. Fedorov, A. A. Kornyshev, Ionic liquids at electrified interfaces, Chem. Rev. 114 (2014) 2978-3036. 
[4] T. Torimoto, T. Tsuda, K.-I. Okazaki, S. Kuwabata, New frontiers in materials science opened by ionic liquids, Adv. Mater. 22 (2010) 11961221.

[5] H. Olivier-Bourbigou, L. Magna, D. Morvan, Ionic liquids and catalysis: Recent progress from knowledge to applications, Appl. Catal., A 373 (2010) 1-56.

[6] M. Z. Bazant, M. S. Kilic, B. D. Storey, A. Ajdari, Towards an understanding of induced-charge electrokinetics at large applied voltages in concentrated solutions, Adv. Colloid Interface Sci. 152 (2009) 48-88.

[7] M. Z. Bazant, B. D. Storey, A. A. Kornyshev, Double layer in ionic liquids: Overscreening versus crowding, Phys. Rev. Lett. 109 (2011) 046102.

[8] J. Vatamanu, D. Bedrov, Capacitive energy storage: Current and future challenges, J. Phys. Chem. Lett. 6 (2015) 3594-3609.

[9] S. Kondrat, A. A. Kornyshev, Pressing a spring: what does it take to maximize the energy storage in nanoporous supercapacitors?, Nanoscale Horiz. 1 (2016) 45-52.

[10] T. Y. Kim, H. W. Lee, M. Stoller, D. R. Dreyer, C. W. Bielawski, R. S. Ruoff, K. S. Suh, High-performance supercapacitors based on poly(ionic liquid)-modified graphene electrodes, ACS NANO 5 (2010) 436-442.

[11] Wan-YuTsai, RongyingLin, S. Murali, L. L. Zhang, J. K. McDonough, R. S. Ruoff, Pierre-LouisTaberna, Y. Gogotsi, P. Simon, Outstanding performance of activated graphene based supercapacitors in ionic liquid electrolyte from 50 to $80{ }^{\circ} \mathrm{c}$, NANO ENERGY 2 (2013) 403-411.

[12] A. A. Kornyshev, Double-layer in ionic liquids: Paradigm change?, J. Phys. Chem. B 111 (2007) 5545-5557.

[13] N. Gavish, A. Yochelis, Theory of phase separation and polarization for pure ionic liquids, J. Phys. Chem. Lett. 7 (2016) 1121-1126.

[14] L. B. Bhuiyan, S. Lamperski, J. Wu, D. Henderson, Monte carlo simulation for the double layer structure of an ionic liquid using a dimer 
model: A comparison with the density functional theory, J. Phys. Chem. B 116 (2012) 10364-10370.

[15] A. Härtel, S. Samin, R. van Roij, Dense ionic fluids confined in planar capacitors: in- and out-of-plane structure from classical density functional theory, J. Phys.: Condens. Matter 28 (2016) 244007.

[16] J. Wu, T. Jiang, D. en Jiang, Z. Jin, D. Henderson, A classical density functional theory for interfacial layering of ionic liquids, Soft Mater 7 (2011) 11222-11231.

[17] M. Trulsson, J. Algotsson, J. Forsman, C. E. Woodward, Differential capacitance of room temperature ionic liquids: The role of dispersion forces, J. Phys. Chem. Lett. 1 (2010) 1191-1195.

[18] M. V. Fedorov, A. A. Kornyshev, Towards understanding the structure and capacitance of electrical double layer in ionic liquids, Electrochim. Acta 53 (2008) 6835-6840.

[19] M. S. Loth, B. Skinner, B. I. Shklovskii, Anomalously large capacitance of an ionic liquid described by the restricted primitive model, Phys. Rev. E 82 (2010) 056102.

[20] J. Vatamanu, O. Borodin, G. D. Smith, Molecular insights into the potential and temperature dependences of the differential capacitance of a room-temperature ionic liquid at graphite electrodes, J. Am. Chem. Soc. 132 (2010) 14825-14833.

[21] Q. Dou, M. L. Sha, H. Y. Fu, G. Z. Wu, Molecular dynamics simulation of the interfacial structure of $\left[C_{(n)} \operatorname{mim}\right]\left[P F_{6}\right]$ adsorbed on a graphite surface: effects of temperature and alkyl chain length, J. Phys.: Condens. Matter 23 (2011) 175001.

[22] J. Vatamanu, L. Xing, W. Li, D. Bedrov, Influence of temperature on the capacitance of ionic liquid electrolytes on charged surfaces, Phys. Chem. Chem. Phys. 16 (2014) 5174-5182.

[23] C. Merlet, B. Rotenberg, P. A. Maddenc, M. Salanne, Computer simulations of ionic liquids at electrochemical interfaces, Phys. Chem. Chem. Phys. 15 (2013) 15781-15792. 
[24] X. Liu, Y. Han, T. Yan, Temperature effects on the capacitance of an imidazolium-based ionic liquid on a graphite electrode: A molecular dynamics simulation, ChemPhysChem 15 (2014) 2503-2509.

[25] M. A. Gebbie, M. Valtiner, X. Banquy, E. T. Fox, W. A. Henderson, J. N. Israelachvili, Ionic liquids behave as dilute electrolyte solutions, PNAS 110 (2013) 9674-9679.

[26] M. A. Gebbie, H. A. Dobbs, M. Valtinerc, J. N. Israelachvili, Longrange electrostatic screening in ionic liquids, PNAS 112 (2015) 74327437 .

[27] A. M. Smith, A. A. Lee, S. Perkin, The electrostatic screening length in concentrated electrolytes increases with concentration, J. Phys. Chem. Lett. 7 (2016) 2157-2163.

[28] F. Endres, N. Borisenko, S. Z. E. Abedin, R. Hayes, R. Atkin, The interface ionic liquid(s)/electrode(s): In situ STM and AFM measurements, Faraday Discuss. 154 (2012) 221-233.

[29] R. Atkin, S. Z. E. Abedin, R. Hayes, L. H. S. Gasparotto, N. Borisenko, F. Endres, AFM and STM studies on the surface interaction of [BMP]TFSA and (EMim]tfsa ionic liquids with $\mathrm{Au}(111)$, J. Chem. Phys. C 113 (2009) 13266-13272.

[30] V. Lockett, R. Sedev, J. Ralston, M. Horne, T. Rodopoulos, Differential capacitance of the electrical double layer in imidazolium-based ionic liquids: Influence of potential, cation size, and temperature, J. Phys. Chem. C 112 (2008) 7486-7495.

[31] V. Lockett, M. Horne, R. Sedev, T. Rodopoulos, J. Ralston, Differential capacitance of the double layer at the electrode/ionic liquids interface, Phys. Chem. Chem. Phys. 12 (2010) 12499-12512.

[32] M. Gnahm, C. Müller, R. Répánszki, T. Pajkossy, D. M. Kolb, The interface between $\mathrm{Au}(100)$ and 1-butyl-3-methyl-imidazoliumhexafluorophosphate, Phys. Chem. Chem. Phys. 13 (2011) 1162711633. 
[33] M. T. Alam, J. Masud, M. M. Islam, T. Okajima, T. Ohsaka, Differential capacitance at $\mathrm{Au}(111)$ in 1-alkyl-3-methylimidazolium tetrafluoroborate based room-temperature ionic liquids, J. Chem. Phys. C 115 (2011) 19797-19804.

[34] F. Silva, C. Gomes, M. Figueiredo, R. Costa, A. Martins, C. M. Pereira, The electrical double layer at the [BMIM] $[\mathrm{PF} 6]$ ionic liquid/electrode interface - effect of temperature on the differential capacitance, J. Electroanal. Chem. 622 (2008) 153-160.

[35] R. Costa, C. M. Pereira, F. Silva, Double layer in room temperature ionic liquids: influence of temperature and ionic size on the differential capacitance and electrocapillary curves, Phys. Chem. Chem. Phys. 12 (2010) 11125-11132.

[36] C. Cannes, H. Cachet, C. Debiemme-Chouvy, C. Deslouis, J. de Sanoit, C. L. Naour, V. A. Zinovyeva, Double layer at $[B \mathrm{u} M \mathrm{e} I \mathrm{~m}]\left[T \mathrm{f}_{2} N\right]$ ionic liquid- $P$ t or $-C$ material interfaces, J. Phys. Chem. C 117 (2013) 2291522925.

[37] M. Drüschler, N. Borisenko, J. Wallauer, C. Winter, B. Huber, F. Endres, B. Roling, New insights into the interface between a singlecrystalline metal electrode and an extremely pure ionic liquid: slow interfacial processes and the influence of temperature on interfacial dynamics, Phys. Chem. Chem. Phys. 14 (2012) 5090-5099.

[38] J. Vatamanu, O. Borodin, D. Bedrov, G. D. Smith, Molecular dynamics simulation study of the interfacial structure and differential capacitance of alkylimidazolium bis(trifluoromethanesulfonyl)imide $\left[\mathrm{C}_{n} \mathrm{mim}\right][t f s i]$ ionic liquids at graphite electrodes, J. Phys. Chem. C 116 (2012) 79407951.

[39] S. Lamperski, C. W. Outhwaite, L. B. Bhuiyan, The electric doublelayer differential capacitance at and near zero surface charge for a restricted primitive model electrolyte, J. Chem. Phys. B 113 (2009) 8925-8929.

[40] J. Vatamanu, O. Borodin, G. D. Smith, Molecular simulations of the electric double layer structure, differential capacitance, and charging 
kinetics for n-methyl-npropylpyrrolidinium bis(fluorosulfonyl)imide at graphite electrodes, J. Chem. Phys. B 115 (2011) 3073-3084.

[41] K. Kirchner, A simulation study of simple ionic liquids near charged walls : the melting of the electric double layers and structural transitions at the interface, Ph.D. thesis, University of Strathclyde, Department of Physics (2013).

[42] M. Holovko, V. Kapko, D. Henderson, D. Boda, On the influence of ionic association on the capacitance of an electrical double layer, Chem. Phys. Lett. 341 (2001) 363-368.

[43] J. Reszko-Zygmunt, S. Sokołowski, D. Henderson, D. Boda, Temperature dependence of the double layer capacitance for the restricted primitive model of an electrolyte solution from a density functional approach, J. Chem. Phys. 112 (2005) 084504.

[44] D. Boda, D. Henderson, The capacitance of the solvent primitive model double layer at low effective temperatures, J. Chem. Phys. 112 (2000) 8934-8938.

[45] D. di Caprio, M. Valiskó, M. Holovko, D. Boda, Anomalous temperature dependence of the differential capacitance in valence asymmetric electrolytes. comparison of monte carlo simulation results and the field theoretical approach, Mol. Phys. 104 (2006) 3777-3786.

[46] D. Boda, D. Henderson, K. Y. Chan, D. T. Wasan, Low temperature anomalies in the properties of the electrochemical interface, Chem. Phys. Lett. 308 (1999) 473-478.

[47] D. di Caprio, J. Stafiej, Z. Borkowska, Anomalous temperature dependence of differential capacity at an uncharged interface with DebyeHückel electrolyte: Field theoretical approach, J. Electroanal. Chem. 582 (2005) 41-49.

[48] D. Boda, D. Henderson, K.-Y. Chan, Monte carlo study of the capacitance of the double layer in a model molten salt, J. Chem. Phys. 110 (1999) 5346-5350. 
[49] M. A. Gebbie, M. Valtiner, X. Banquy, W. A. Henderson, J. N. Israelachvilia, Reply to perkin et al.: Experimental observations demonstrate that ionic liquids form both bound (Stern) and diffuse electric double layers, PNAS 110 (2013) E4122.

[50] S. Perkin, M. Salanne, P. Madden, R. Lynden-Bell, Is a Stern and diffuse layer model appropriate to ionic liquids at surfaces?, PNAS 110 (2013) E4121.

[51] A. A. Lee, D. Vella, S. Perkin, A. Goriely, Are room-temperature ionic liquids dilute electrolytes?, J. Phys. Chem. Lett. 6 (2015) 159-163.

[52] K. Ma, J. Forsman, C. E. Woodward, Influence of ion pairing in ionic liquids on electrical double layer structures and surface force using classical density functional approach, J. Chem. Phys. 142 (2015) 174704.

[53] Z. A. H. Goodwin, A. A. Kornyshev, Underscreening, overscreening and double-layer capacitance, Electrochem. Commun. 82 (2017) 129133.

[54] R. M. Adar, T. Markovich, D. Andelman, Bjerrum pairs in ionic solutions: A poisson-boltzmann approach, J. Chem. Phys. 146 (2017) 194904.

[55] O. Hollóczki, F. Malberg, T. Welton, B. Kirchner, On the origin of ionicity in ionic liquids. ion pairing versus charge transfer, Phys. Chem. Chem. Phys. 16 (2014) 16880-16890.

[56] K. R. Harris, Can the transport properties of molten salts and ionic liquids be used to determine ion association?, J. Chem. Phys. B 120 (2016) 12135-12147.

[57] Y. Zhang, E. J. Maginn, Direct correlation between ionic liquid transport properties and ion pair lifetimes: A molecular dynamics study, J. Phys. Chem. Lett. 6 (2015) 700-705.

[58] M. Sha, H. Dong, F. Luo, Z. Tang, G. Zhu, G. Wu, Dilute or concentrated electrolyte solutions? insight from ionic liquid/water electrolytes, J. Phys. Chem. Lett. 6 (2015) 3713-3720. 
[59] H. K. Kashyap, H. V. R. Annapureddy, F. O. Raineri, C. J. Margulis, How is charge transport different in ionic liquids and electrolyte solutions?, J. Phys. Chem. B 115 (2011) 13212-13221.

[60] M. A. Gebbie, A. M. Smith, H. A. Dobbs, A. A. Lee, G. G. Warr, X. Banquy, M. Valtiner, M. W. Rutland, J. N. Israelachvili, S. Perkin, R. Atkin, Long range electrostatic forces in ionic liquids, Chem. Commun. 53 (2017) 1214-1224.

[61] B. Kirchner, F. Malberg, D. S. Firaha, O. Hollóczki, Ion pairing in ionic liquids, J. Phys.: Condens. Matter 27 (2015) 463002.

[62] R. Hayes, G. G. Warr, R. Atkin, Structure and nanostructure in ionic liquids, Chem. Rev. 115 (2015) 6357-6426.

[63] W. Zhao, F. Leroy, B. Heggen, S. Zahn, B. Kirchner, S. B. F. MüllerPlathe, Are there stable ion-pairs in room-temperature ionic liquids? molecular dynamics simulations of 1-n-butyl-3-methylimidazolium hexafluorophosphate, J. Am. Chem. Soc. 131 (2009) 15825-15833.

[64] M. Sha, G. Wu, Y. Liu, Z. Tang, H. Fang, Drastic phase transition in ionic liquid [Dmim] $[\mathrm{Cl}]$ confined between graphite walls: New phase formation, J. Phys. Chem. C 113 (2009) 4618-4622.

[65] M. Sha, G. Wu, H. Fang, G. Zhu, Y. Liu, Liquid-to-solid phase transition of a 1,3-dimethylimidazolium chloride ionic liquid monolayer confined between graphite walls, J. Chem. Phys. C 112 (2008) 1858418587.

[66] S. Perkin, Ionic liquids in confined geometries, Phys. Chem. Chem. Phys. 14 (2012) 5052-5062.

[67] M. S. Kilic, M. Z. Bazant, A. Ajdari, Steric effects in the dynamics of electrolytes at large applied voltages. i. double-layer charging, Phys. Rev. E 75 (2007) 021502.

[68] J. Zwanikken, R. van Roij, Inflation of the screening length induced by Bjerrum pairs, J. Phys.: Condens. Matter 21 (2009) 424102.

[69] Z. Gu, J. F. Brennecke, Volume expansivities and isothermal compressibilities of imidazolium and pyridinium-based ionic liquids, J. Chem. Eng. Data 47 (2002) 339-345. 
[70] S. Aparicio, R. Alcalde, B. Garć, J. M. Leal, High-pressure study of the methylsulfate and tosylate imidazolium ionic liquids, J. Phys. Chem. B 113 (2009) 5593-5606.

[71] M. Kanakubo, K. R. Harris, Density of 1-butyl-3-methylimidazolium bis(trifluoromethanesulfonyl)amide and 1-hexyl-3- methylimidazolium bis(trifluoromethanesulfonyl)amide over an extended pressure range up to $250 \mathrm{MPa}$, J. Chem. Eng. Data 60 (2015) 1408-1418.

[72] R. Taguchi, H. Machida, Y. Sato, R. L. Smith, High-pressure densities of 1-alkyl-3-methylimidazolium hexafluorophosphates and 1-alkyl3-methylimidazolium tetrafluoroborates at temperatures from (313 to 473) K and at pressures up to $200 \mathrm{MPa}$, J. Chem. Eng. Data 54 (2009) $22-27$.

[73] H. Machida, Y. Sato, R. L. S. Jr., Pressure-volume-temperature (PVT) measurements of ionic liquids $\left(\left[\mathrm{bmim}^{+}\right]\left[\mathrm{PF}_{6}^{-}\right],\left[\mathrm{bmim}^{+}\right]\left[\mathrm{BF}_{4}^{-}\right]\right.$, $\left.\left[\mathrm{bmim}^{+}\right]\left[\mathrm{OcSO}_{4}^{-}\right]\right)$and analysis with the sanchez-lacombe equation of state, Fluid Phase Equilibria 264 (2008) 147-155.

[74] Y. Yua, D. Bejanc, R. Krause-Rehberg, Free volume investigation of imidazolium ionic liquids from positron lifetime spectroscopy, Fluid Phase Equilibria 363 (2014) 48-54.

[75] Y. Yu, W. Beichel, G. Dlubek, R. Krause-Rehberg, M. Paluch, J. Pionteck, D. Pfefferkorn, S. Bulut, C. Friedrich, N. Pogodina, I. Krossing, Free volume and phase transitions of 1-butyl-3-methylimidazolium based ionic liquids from positron lifetime spectroscopy, Phys. Chem. Chem. Phys. 14 (2012) 6859-6868.

[76] W. Beichel, Y. Yu, G. Dlubek, R. Krause-Rehberg, J. Pionteck, D. Pfefferkorn, S. Bulut, D. Bejan, C. Friedrich, I. Krossing, Free volume in ionic liquids: a connection of experimentally accessible observables from PALS and PVT experiments with the molecular structure from XRD data, Phys. Chem. Chem. Phys. 15 (2013) 8821-8830.

[77] G. Dlubek, Y. Yu, R. Krause-Rehberg, W. Beichel, S. Bulut, N. Pogodina, I. Krossing, C. Friedrich, Free volume in imidazolium triflimide $\left[\mathrm{C}_{3} \mathrm{MIM}\right]\left[\mathrm{NTf}_{2}\right]$ ionic liquid from positron lifetime: Amorphous, crystalline, and liquid states, J. Phys. Chem. 133 (2010) 124502. 
[78] N. C. Forero-Martinez, R. Cortes-Huerto, P. Ballone, The glass transition and the distribution of voids in room-temperature ionic liquids: A molecular dynamics study, J. Chem. Phys. 136 (2012) 204510.

[79] A. P. Abbott, Application of hole theory to the viscosity of ionic and molecular liquids, ChemPhysChem 5 (2004) 1242-1246.

[80] A. P. Abbott, Model for the conductivity of ionic liquids based on an infinite dilution of holes, ChemPhysChem 6 (2005) 2502-2505.

[81] I. Krossing, J. M. Slattery, Semi-empirical methods to predict the physical properties of ionic liquids: An overview of recent developments, Z. Phys. Chem. 220 (2006) 1343-1359.

[82] R. Fürth, On the theory of the liquid state i. the statistical treatment of the thermodynamics of liquids by the theory of holes, Proc. Cambridge Phil. Soc. 37 (1941) 252-275.

[83] R. Fürth, Proc. Cambridge Phil. Soc. 37 (1941) 276-280.

[84] R. Fürth, On the theory of the liquid state iii. the hole theory of viscous flow of liquids, Proc. Cambridge Phil. Soc. 37 (1941) 281-290.

[85] D. McQuarrie, Statistical Mechanics, 1st Edition, Harper and Row, New York, 1976.

[86] Z. A. H. Goodwin, G. Feng, A. A. Kornyshev, Mean-field theory of electrical double layer in ionic liquids with account of short-range correlations, Electrochim. Acta 225 (2017) 190-196.

[87] N. Gavish, D. Elad, A. Yochelis, From solvent free to dilute electrolytes: A unified continuum approach (2017) arXiv preprint arXiv:1707.06940.

[88] M. V. Fedorov, A. A. Kornyshev, Ionic liquid near a charged wall: Structure and capacitance of electrical double layer, J. Phys. Chem. B 112 (2008) 11868-11872.

[89] N. Georgi, A. A. Kornyshev, M. V. Fedorov, The anatomy of the double layer and capacitance in ionic liquids with anisotropic ions: Electrostriction vs. lattice saturation, J. Electroanal. Chem. 649 (2010) 261-267. 
[90] T. Fang, A. Konar, H. Xing, D. Jena, Carrier statistics and quantum capacitance of graphene sheets and ribbons, Appl. Phys. Lett. 91 (2007) 092109.

[91] H. Xu, Z. Zhang, L.-M. Peng, Measurements and microscopic model of quantum capacitance in graphene, Appl. Phys. Lett. 98 (2011) 133122.

[92] E. Paek, A. J. Pak, G. S. Hwang, A computational study of the interfacial structure and capacitance of graphene in $[\mathrm{BMIM}]\left[\mathrm{PF}_{6}\right]$ ionic liquid, J. Electrochem. Soc. 160 (2012) A1 - A10.

[93] A. A. Kornyshev, N. B. Luque, W. Schmickler, Differential capacitance of ionic liquid interface with graphite: the story of two double layers, J Solid State Electrochem. 18 (2014) 1345-1349.

[94] A. J. Pak, E. Paekw, G. S. Hwang, Relative contributions of quantum and double layer capacitance to the supercapacitor performance of carbon nanotubes in an ionic liquid, Phys. Chem. Chem. Phys. 15 (2013) 19741-19747.

[95] J. Vatamanu, X. Ni, F. Liu, D. Bedrov, Tailoring graphene-based electrodes from semiconducting to metallic to increase the energy density in supercapacitors, Nanotechnology 26 (2015) 464004.

[96] D. V. der Spoel, E. Lindahl, B. Hess, t.G.d. team, GROMACS User Manual version 4.6.2., University of Groningen, Royal Institute of Technology and Uppsala University (2013).

[97] L. Verlet, Computer "experiments" on classical fluids. i. thermodynamical properties of Lennard-Jones molecules, Phys. Rev. 159 (1967) 98-103.

[98] B. Hess, H. Bekker, H. Berendsen, J. Fraaije, LINCS: A linear constraint solver for molecular simulations, J. Comput. Chem. 18 (1997) $1463-1472$.

[99] Y. Zhang, E. Maginn, A simple AIMD approach to derive atomic charges for condensed phase simulation of ionic liquids, J. Chem. Phys. B 116 (2012) 10036-10048. 
[100] C. Schroder, Comparing reduced partial charge models with polarizable simulations of ionic liquids, Phys. Chem. Chem. Phys. 14 (2012) 3089102.

[101] W. Cornell, P. Cieplak, C. I. Bayly, I. R. Gould, K. M. Merz, D. M. Ferguson, D. C. Spellmeyer, T. Fox, J. W. Caldwell, P. Kollman, A second generation force field for the simulation of proteins, nucleic acids, and organic molecules, J. Am. Chem. Soc. 117 (1995) 5179-5197.

[102] T. Darden, D. York, L. Pedersen, Particle mesh ewald: An NlnN method for ewald sums in large systems, J. Chem. Phys. 98 (1993) 10089-10092.

[103] I. C. Yeh, M. Berkowitz, Ewald summation for systems with slab geometry, J. Chem. Phys. 111 (1999) 3155-3162.

[104] J. Vatamanu, L. Cao, O. Borodin, D. Bedrov, G. Smith, On the influence of surface topography on the electric double layer structure and differential capacitance of graphite/ionic liquid interfaces, J. Phys. Chem. Lett. 2 (2011) 2267-2272.

[105] B. Qiao, X. Zhao, D. Yue, L. Zhang, S. Wu, A combined experiment and molecular dynamics simulation study of hydrogen bonds and free volume in nitrile-butadiene rubber/hindered phenol damping mixtures, J. Mater. Chem. 22 (2012) 12339-12348.

[106] A. Bondi, van der waals volumes and radii, J. Phys. Chem. 68 (1964) $441-451$. 